\title{
Uremic tumoral calcinosis: A case report
}

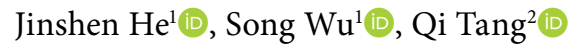 \\ ${ }^{1}$ Department of Orthopedic Surgery, Third Xiangya Hospital of Central South University, Changsha, China \\ ${ }^{2}$ Department of Rheumatology and Immunology, Second Xiangya Hospital of Central South University, Changsha, China
}

Tumoral calcinosis, first termed by Inclan et al., ${ }^{1}$ is a rare condition characterized by massive metastatic calcification around joints, which presents as single or multiple superficial or deep hard caseous nodules located periarticularly. The common symptoms of tumoral calcinosis are joint stiffness, joint functional impairment, and neurovascular compression. In the present case, the nodules were situated around the shoulder and wrist with no other symptoms. In particular, we, for the first time, report calcium oxalate crystal imaging in the mass via polarized microscopy.

A 27-year-old man complained of gradually enlarging and tender masses over the left shoulder for one year. He had end-stage renal disease and was receiving maintenance hemodialysis for five years. Physical examination revealed subcutaneous and fluctuant masses around the left shoulder (Figure 1a). There was no deformity in the neck. The neck joint had a normal range of motion (ROM). Spurling test result was negative. The mass around the shoulder was soft. The Tinel's sign was negative. The ROM of the left shoulder joint was significantly limited. The muscle strength, muscle tension, and sensation of the left upper limb were normal. Laboratory examinations showed normal serum calcium
(2.2 $\mathrm{mmoL} / \mathrm{L}$; reference: 2.2-2.7 $\mathrm{mmoL} / \mathrm{L})$, elevated serum phosphorus $(1.86 \mathrm{mmoL} / \mathrm{L}$; reference: $0.85-1.51 \mathrm{mmoL} / \mathrm{L})$, and elevated parathyroid hormone levels $(1157 \mathrm{pg} / \mathrm{mL}$; reference: $15-65 \mathrm{pg} / \mathrm{mL})$. X-ray showed speckled amorphous, globular calcifications around the shoulder (Figure 1b). Magnetic resonance imaging demonstrated a heterogeneous signal intensity with cystic and calcific components, but without evidence of humerus erosion on T2-weighted imaging (Figure 1c). Biopsy revealed calcifications surrounded by histiocytic and giant cells reaction. Needle aspiration yielded a viscous liquid containing white grits (Figure 1d), and the majority of calcium oxalate crystals were identified on polarized microscopy and infrared spectroscopy (Figure 1e). Neck ultrasonography revealed hyperplasia of parathyroid glands. The patient was diagnosed with tumoral calcinosis. Dietary restrictions and subtotal parathyroidectomy were applied to the patient, surgical excision of the masses was not performed for probable recurrence. ${ }^{2}$ The patient was on the waiting list for kidney transplantation as the etiological treatment, which could decrease the mass with balanced calcium-phosphate metabolism. ${ }^{3}$ The case had

Received: December 07, 2020 Accepted: January 07, 2021 Published online: February 08, 2021

Correspondence: Qi Tang, MD. Department of Rheumatology and Immunology, Second Xiangya Hospital of Central South University, 410000 Changsha, China. Tel: +8613517470997 e-mail: tangqi8824@csu.edu.cn 

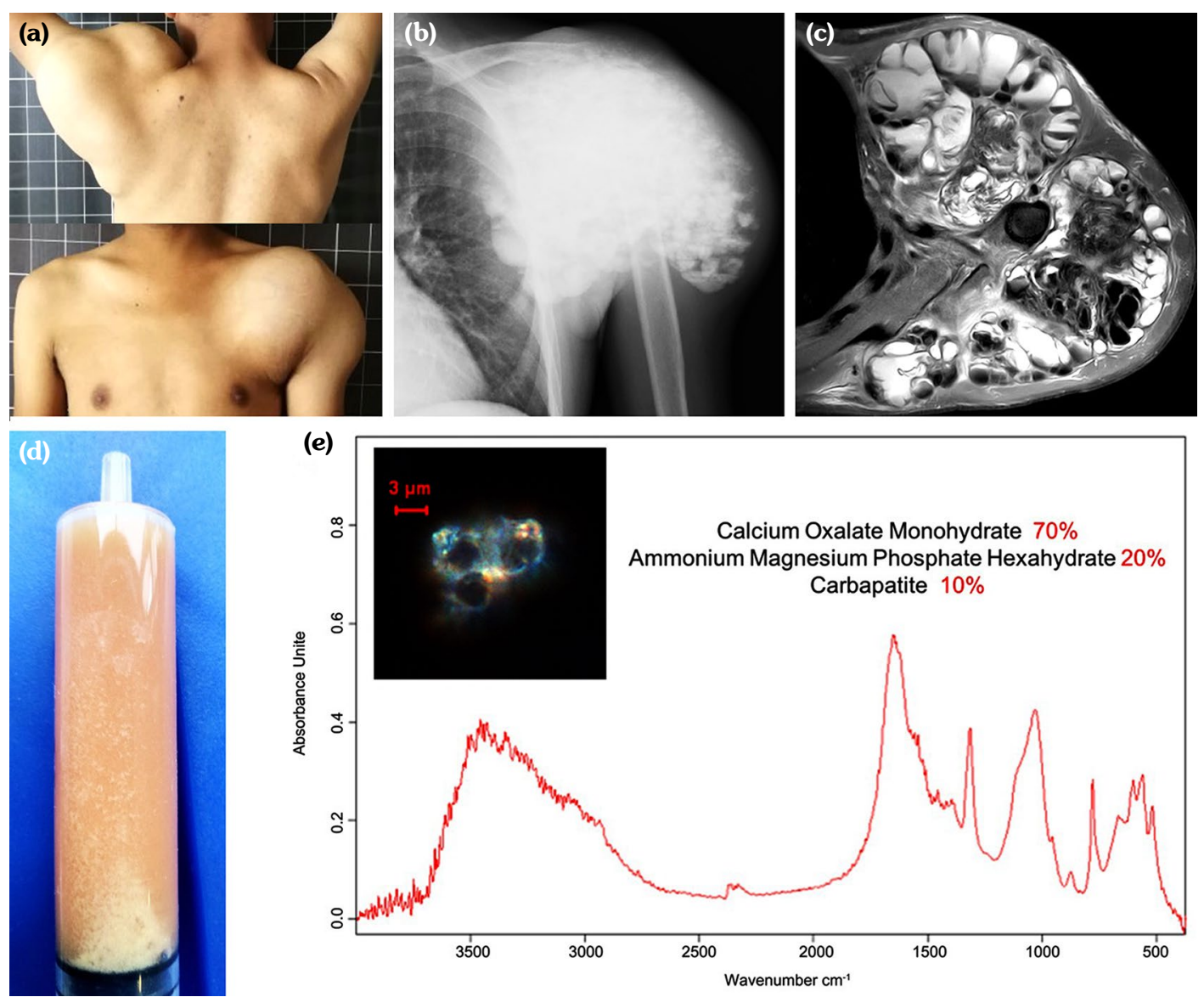

Figure 1. Examinations of the patient. (a) Physical examination showing a massive, fluctuant, subcutaneous mass covering the left shoulder. (b) X-ray showing speckled amorphous, globular calcifications around the shoulder. (c) Magnetic resonance imaging showing a heterogeneous signal intensity around shoulder with cystic and calcific components, but without humerus erosive evidence. (d) Needle aspiration showing a viscous liquid containing white grits, with the majority of calcium oxalate crystals identified on (e) polarized microscopy and infrared spectroscopy.

no signs of mass aggravation or new lesions at 36 months of follow-up.

A written informed consent was obtained from the patient.

Tumoral calcinosis may be primary or secondary. The primary form implicates autosomal recessive mutations of three genes involved in phosphate metabolism: fibroblast growth factor 23 (FGF23), GalNAc transferase 3 (GALNT3), and Klotho (KL). ${ }^{4}$ Secondary forms are due to either phosphocalcic metabolism abnormality or other abnormalities. In the present case, tumoral calcinosis was a complication of terminal chronic kidney failure under dialysis, known as uremic tumoral calcinosis (UTC).
In kidney failure, a high calcium-phosphate product with coexistent hyperparathyroidism leads to calcium-phosphate salts precipitation in the periarticular soft tissues surrounding weight-bearing or overused joints. ${ }^{5}$ Nearly all patients with UTC have hyperphosphatemia, regardless of serum calcium levels. Many evidences were obtained in this end-stage renal disease patient with confirmed UTC: hyperphosphatemia and hyperparathyroidism; radiology showing cystic calcification; needle aspiration showing a calcareous material; pathology showing calcification infiltration of the surrounding area with giant cells; and polarized microscopy and infrared spectroscopy showing the majority of calcium oxalate crystals. 
In conclusion, we, for the first time, report the crystal imaging in UTC. The UTC nodules can be differentiated from gout nodules through crystal imaging. Monosodium urate crystals, ${ }^{6}$ not calcium oxalate crystals, are identified on polarized microscopical examination of gouty patients.

\section{Declaration of conflicting interests}

The authors declared no conflicts of interest with respect to the authorship and/or publication of this article.

\section{Funding}

The study is supported by the National Natural Science Foundation of China (No. 81701552, No. 81802208). The funds were used for polarized microscopy and infrared spectroscopy.

\section{REFERENCES}

1. Inclan A, Leon PP, Camejo M. Tumoral calcinosis. J Am Med Ass 1943;121:490-5.

2. Rao N, Crail S. Images in clinical medicine. Metastatic calcification and long-term hemodialysis. $\mathrm{N}$ Engl $\mathrm{J}$ Med 2013;368:2415.

3. Megaloikonomos PD, Mavrogenis AF, Panagopoulos GN, Kontogeorgakos VA. Tumoral calcinosis of the shoulder. Lancet Oncol 2017;18:e126.

4. McClatchie S, Bremner AD. Tumoral calcinosis--an unrecognized disease. Br Med J 1969;1:153-5.

5. Ibrahim Montasser D, Issouani J, Hassani M, Kabbaj D. Tumoral calcinosis: Diffuse multifocal form in hemodialysis patients. Two case reports. Orthop Traumatol Surg Res 2017;103:815-20.

6. Cheema U. Images in clinical medicine. Gout nodulosis. N Engl J Med 2011;365:e23. 